\title{
A Simple Region Descriptor based on Object Area per Scan Line
}

\author{
R. Deepak Kumar \\ Student \\ School of Interdisciplinary Science \\ and Technology \\ I2IT, Pune
}

\author{
K.Ramareddy \\ Student \\ Embedded System Design \\ I2IT, Pune
}

\author{
B.Madhusudan Rao \\ Senior Research Assistant \\ Embedded System Design \\ I2IT, Pune
}

\begin{abstract}
In the field of image processing, identifying object is based on appropriately chosen descriptors. The proper choice of descriptors in pattern recognition is the most sensitive criteria as small misjudgments may lead to wrong identification. There have been several algorithms proposed and worked in this field. Here, the idea is to identify the objects in an uncomplicated method while being computationally efficient. This paper is based on identifying the patterns of objects / polygons based on the recording area of the objects per line scan. The descriptors here are invariant to translation and become invariant to scaling after normalization. Here the objects considered are regular polygons in various background conditions. In order to reduce the noise, after segmentation by thresholding along with labeling and area filtering is done. Along with polygon identification, descriptors for all the numbers are also shown. In order to identify the objects, the average magnitude difference function (AMDF) is applied to each characteristic curve. This paper also shows that though AMDF is a dissimilarity measure, it works better here than auto correlation function $(\mathrm{ACF})$, which is a similarity measure.
\end{abstract}

\section{General Terms}

Pattern Recognition, Region based descriptors, AMDF, ACF.

\section{Keywords}

Area Filtering, Object area per scan line, AMDF, ACF.

\section{INTRODUCTION}

Pattern recognition of any object is one of the most important applications of image processing. The descriptors of the object characteristics/patterns must be congruent, invariant to noise, scale, rotation and translation and must define a complete set [1]. Lot of research is being done over this topic. The simplest and earliest approaches to pattern recognition are based on template matching. Though template matching has its own disadvantages [2], it is considered to be simple yet effective way of matching objects.

In general, characterization of objects are split into two methods, region and contour based. Using the shape based techniques; human faces are recognized based on Fourier descriptors using Neural Networks [3]. Underwater objects are described based on wavelet packets and Fourier descriptors [4].However, Fourier descriptor fails in determining shape information of object's corner. Though several shape signatures have been formed [5] for Fourier description, still ideal is yet to be described. Moreover, due to its mathematical complexity it is very tough to implement it in hardware. This kind of problem prevails in Hough transforms too. Further, the generalized Hough transform possess translational invariance but not scale or rotational invariance [6]. Recently, dynamic programming (DP) has been adopted in order to achieve a high accuracy rate using shape boundaries. Despite of this, the DP-based techniques suffer from being computationally expensive [5]

A region usually describes contents (or interior points) which are surrounded by a boundary. Region description can be done based on area, perimeter, etc or based on its moments. The first commercial product for automated biomedical imaging was to classify human white blood cells. One approach was to recognize it based on the area and perimeter [7]. For many other applications, these parameters are not enough. Moment based pattern recognition, though computationally intensive, are very useful in hand gesture recognition [8]. Affine Moment invariant technique is used to recognize characters, independent of size, slant and other variants [9]. The drawback of regular moments is the redundant information in the moments, since the bases are not orthogonal and high-order moments are sensitive to noise [10]. Here the idea is to obtain a region based descriptor which is computationally fast and easy to describe.

In this paper, further, section 2 describes the image segmentation which is common for complete set of images. Section 3 is a detail explanation of how the objects are described. Section 4 contains results of the techniques being used.

\section{PRE PROCESSING AND LOW LEVEL IMAGE SEGMENTATION}

In order to segment the object from image, preprocessing of the image has to be done. Thresholding an image into proper object and background is quite essential. Here, the Otsu's thresholding [11] algorithm is applied. Labeling various objects in the image is done in order to figure out each object and further each object's properties. Various labeling techniques [12] are prevails. Still, the algorithm specified by Haralick, Robert M., and Linda G. Shapiro [13] is employed.

Image segmentation is a post-processing technique which partitions image to certain regions based on characteristics or features. The gray scale image is converted into a binary image by thresholding based on Otsu's algorithm. The labeling of object is done in order the name the objects. There are images which consist of noise due to various back ground conditions. The backgrounds in general are a grass, shadow, mud, etc. Hence after thresholding, majority of image contains small blobs and very few images contain small holes (pepper noise) and unfilled gaps (salt noise). Morphological operators can be used to remove this noise [14]. But for various images different structure element of various 
sizes had to be used, hence it is not feasible. Thus area filtering is done.

The definition of area here is number of object pixels within the object boundary. Here the labeled object whose area is less than certain value (15 percent of area of the object) is removed and those object area which is greater than that value is retained. Sample images are shown in figure $1(a, b, c)$ shows the gray scale images. It can be seen that all three images are different from each other with respect to scaling, lighting conditions and object translation. Figure $2(\mathrm{a}, \mathrm{b}, \mathrm{c})$ shows the images which are obtained after thresholding, which contains noise elements. Figure $3(a, b, c)$ are images after area filtering.
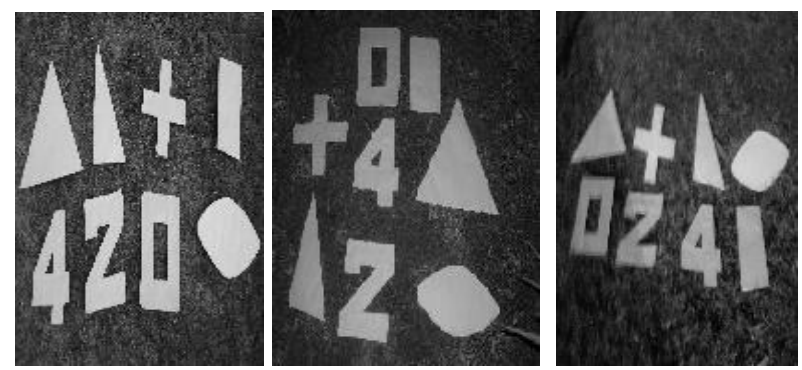

Fig 1.a, 1.b 1.c: Various gray scale images of polygons
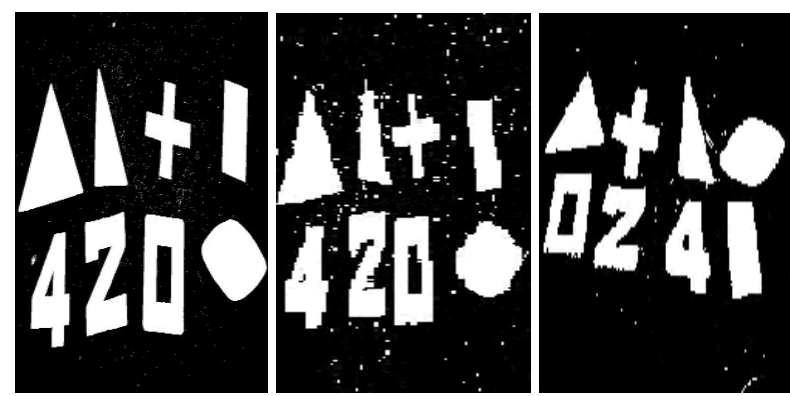

Figure 2.a, 2.b, 2.c: Binary Images after Otsu's thresholding.
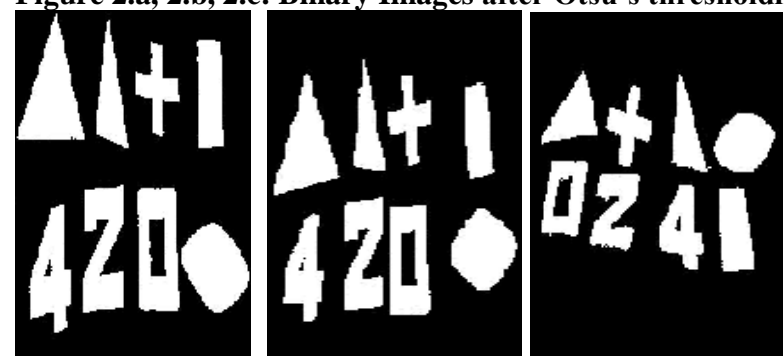

Figure 3.a, 3.b, 3.c: Noise being removed by area filtering.

\section{OBJECT IDENTIFICATION}

When all the noise factors are removed, it is now essential to describe the objects and identify them. Here, the idea is to identify the objects using simple descriptors in region based technique. The descriptors are obtained by scanning each line in the image and obtaining the area of object in the particular line scan. These details are stored in a data base which consists of number of objects and its area under particular line scan.

\subsection{Generation of characteristic curve}

A characteristic curve is defined as a visual representation of the relations between certain quantities plotted with reference to a set of axes. The characteristic curve or descriptors are formed by counting number of pixels of the same labels in each line scan. A database which consists of object's area found per scan is then is then normalized and total scan line is scaled. The number of line scan is scaled between 1 to 100 and the object area per scan line is normalized between 0 to 1 . The graph is plotted based on the table and it is found that the each object descriptors are different from each other. The combined graph for various polygons is shown in the figure $3(\mathrm{a}-\mathrm{h})$.
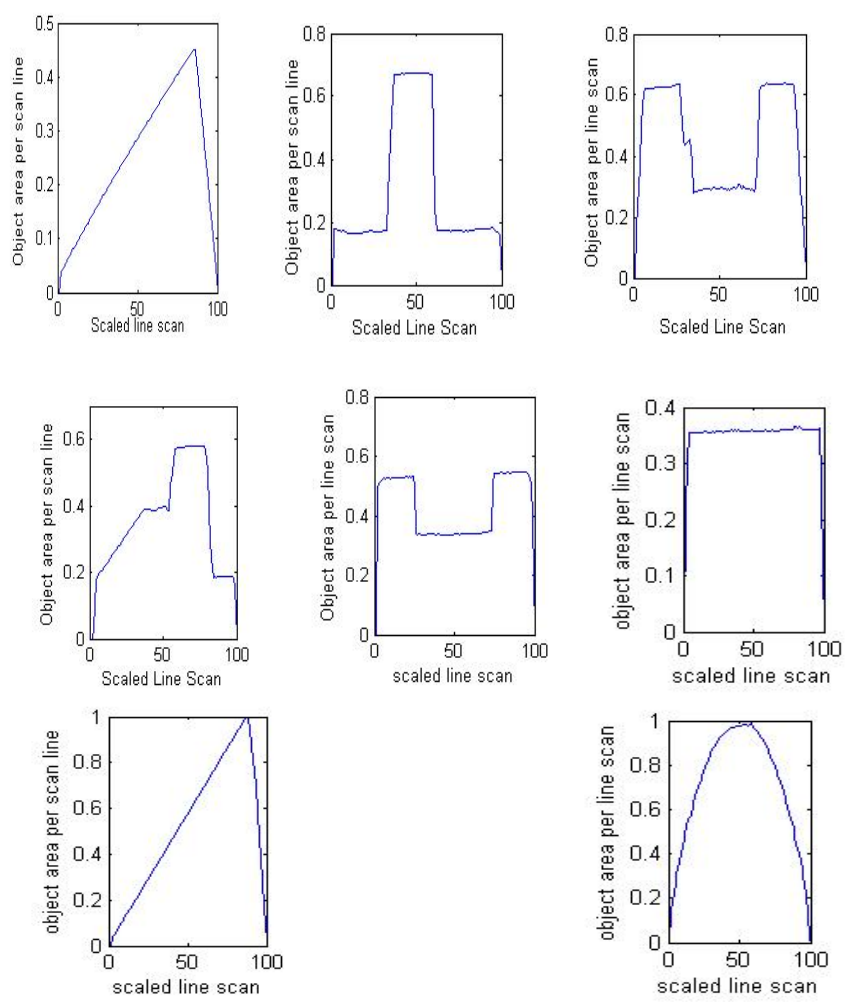

Figure 3 (a - h) (from top left and page 2 right bottom): Characteristic curves for the polygons (right triangle, symbol plus, number two, number four ,number zero, square, equilateral triangle ellipse)

Figure 3 (a-h) shows the characteristic curve for the objects taken. It is noted that descriptor for equilateral triangle is different from right triangle. Its mathematical justification is given in next section. Even visually they are different. This is the case with all other descriptors too. These graphs are similar for around 40-50 images.

This is carried out on all the numbers as an offline work. The descriptor for each number is found to be unique. The image of the numbers taken for finding descriptor is shown in figure 4. 


\section{9}

Figure 4: Image of the numbers.

The descriptors of each number are shown in figure 5(a-i).

Even here, each descriptor is unique.
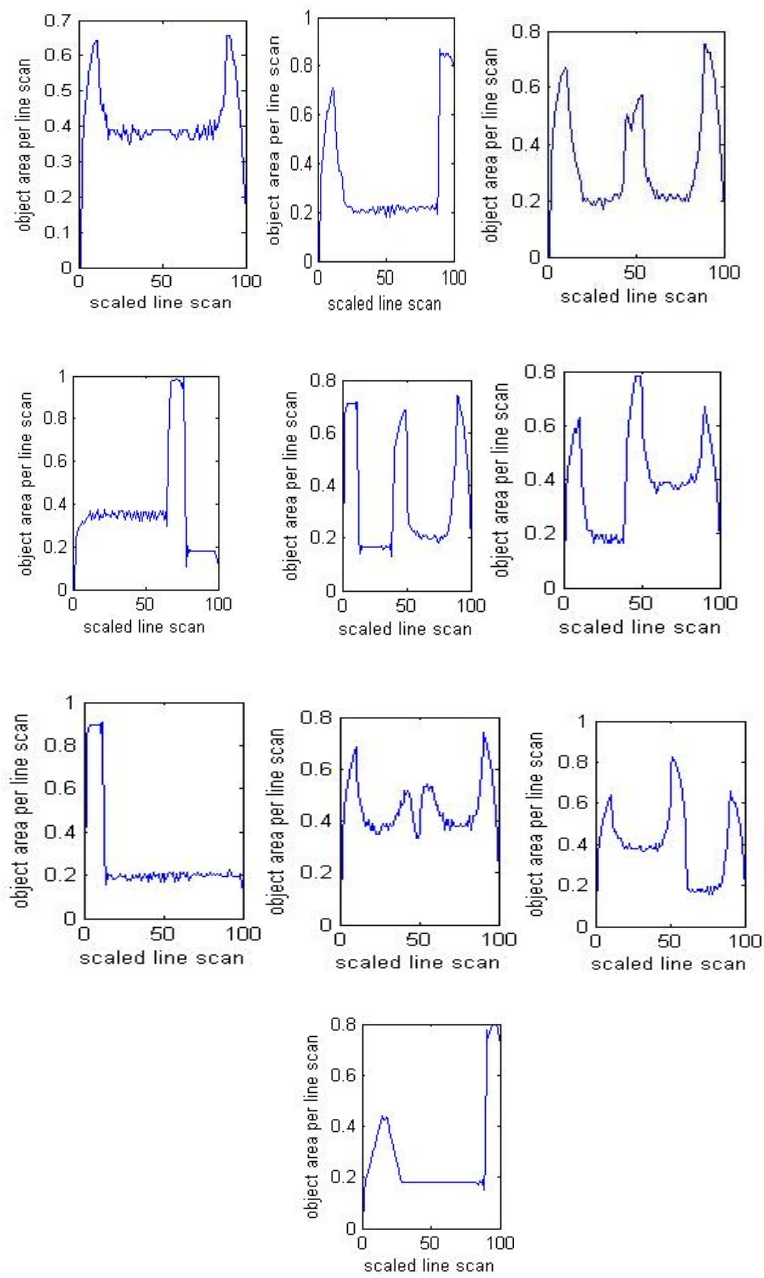

Figure 5(a - i): Characteristic curves for the numbers 0 to 9.

\subsection{Pattern Matching}

Pattern matching is the act of checking for the presence of the constituents (descriptors) of a given pattern. The descriptors are found to be unique as shown in the figure 4 and figure 5 . Since the descriptor here are scaled, the original object cannot be retraced unlike inverse Fourier transform or inverse wavelet transform for Fourier and wavelet descriptors. Here, the 2-D object has now 1-D signal type descriptor. Hence, pattern matching is done based on two standard methods, namely, Auto Correlation Function (ACF) and Average Magnitude Difference Function (AMDF).

\subsubsection{Auto Correlation Function}

ACF is a similarity measure of two curves or signals. It is given as

$$
r[k]=\sum_{i=0}^{N-1} f_{r e f}[i] f_{o b j}[i+k]
$$

where $f_{\text {ref }}$ is values of reference image and $f_{o b j}$ is current object of the image. The coefficient $\mathrm{r}[0](\mathrm{k}=0)$ which gives the maximum value(ideally 1 here) is chosen. This is shown in below equation.

$$
r[0]=\sum_{i=0}^{N-1} f_{r e f}[i] f_{o b j}[i]>\sum_{i=0, k>0}^{N-1} f_{r e f}[i] f_{o b j}[i+k]
$$

It is then the curve or signals are said to be perfectly matched. Here, when ACF coefficient is taken between each curve of same objects and the table is formed, it is found that in some rows the diagonal element is less than the values of that row (which shouldn't be the case). This is because the magnitude of each curve, though normalized is not constant, for example, the normalized area values of the ellipse descriptor is larger for many scaled values than that of a square descriptor, which leads to improper measurement. When ACF is computed, the coefficient between the squares is less than that of coefficients between ellipse and square. Hence, this technique fails, particularly for this application. Hence, a dissimilarity measure with magnitude features, AMDF had to be considered.

\begin{tabular}{|l|l|l|l|l|l|l|l|l|}
\hline & $\mathbf{1}$ & $\mathbf{2}$ & $\mathbf{3}$ & $\mathbf{4}$ & $\mathbf{5}$ & $\mathbf{6}$ & $\mathbf{7}$ & $\mathbf{8}$ \\
\hline $\mathbf{1}$ & 0.36 & 0.18 & 0.39 & 0.77 & 0.15 & 0.06 & 0.84 & 0.52 \\
\hline $\mathbf{2}$ & 0.18 & 0.23 & 0.57 & 0.12 & 0.67 & 0.35 & 0.18 & 0.99 \\
\hline $\mathbf{3}$ & 0.39 & 0.57 & 0.67 & 0.42 & 0.28 & 0.10 & 0.48 & 0.75 \\
\hline $\mathbf{4}$ & 0.77 & 0.12 & 0.42 & 0.76 & 0.16 & 0.08 & 0.83 & 0.54 \\
\hline $\mathbf{5}$ & 0.15 & 0.67 & 0.28 & 0.14 & 0.36 & 0.99 & 0.17 & 0.25 \\
\hline $\mathbf{6}$ & 0.06 & 0.35 & 0.10 & 0.08 & 0.99 & 0.38 & 0.06 & 0.66 \\
\hline $\mathbf{7}$ & 0.84 & 0.18 & 0.48 & 0.83 & 0.17 & 0.06 & 0.14 & 0.19 \\
\hline $\mathbf{8}$ & 0.52 & 0.99 & 0.75 & 0.54 & 0.25 & 0.66 & 0.19 & 0.52 \\
\hline
\end{tabular}

Table 1: ACF coefficients for the polygon descriptors

\subsubsection{Average Magnitude Difference Function}

As stated above, Average Magnitude Difference function is a dissimilarity measure. It is given as

$$
r[k]=\frac{1}{N} \sum_{i=0}^{N-1} f_{r e f}[i]-f_{o b j}[i+k]
$$

The coefficient $\mathrm{r}[0](\mathrm{k}=0)$ is only considered here too as

$$
r[0]=\sum_{i=0}^{N-1} f_{r e f}[i]-f_{o b j}[i]<\sum_{i=0, k>0}^{N-1} f_{r e f}[i]-f_{o b j}[i+k]
$$

Here the absolute value of the difference between the two descriptors is taken. The table is given for the AMDF measure. 


\begin{tabular}{|l|l|l|l|l|l|l|l|l|}
\hline & $\mathbf{1}$ & $\mathbf{2}$ & $\mathbf{3}$ & $\mathbf{4}$ & $\mathbf{5}$ & $\mathbf{6}$ & $\mathbf{7}$ & $\mathbf{8}$ \\
\hline $\mathbf{1}$ & 0.00 & 0.38 & 0.22 & 0.20 & 0.10 & 0.32 & 0.07 & 0.14 \\
\hline $\mathbf{2}$ & 0.38 & 0.00 & 0.11 & 0.10 & 0.19 & 0.18 & 0.20 & 0.10 \\
\hline $\mathbf{3}$ & 0.22 & 0.11 & 0.00 & 0.13 & 0.11 & 0.21 & 0.13 & 0.43 \\
\hline $\mathbf{4}$ & 0.20 & 0.10 & 0.13 & 0.00 & 0.47 & 0.63 & 0.17 & 0.13 \\
\hline $\mathbf{5}$ & 0.10 & 0.19 & 0.11 & 0.47 & 0.00 & 0.27 & 0.43 & 0.20 \\
\hline $\mathbf{6}$ & 0.32 & 0.18 & 0.21 & 0.63 & 0.27 & 0.00 & 0.32 & 0.11 \\
\hline $\mathbf{7}$ & 0.07 & 0.20 & 0.13 & 0.17 & 0.43 & 0.32 & 0.00 & 0.27 \\
\hline $\mathbf{8}$ & 0.14 & 0.10 & 0.43 & 0.13 & 0.20 & 0.11 & 0.27 & 0.00 \\
\hline
\end{tabular}

Table 2: AMDF co-efficient of polygon descriptors

The table is shown for the ideal condition. The numbers from 1 to 8 (in both the tables) are the labels of all the objects. The diagonal elements which must be zero for AMDF (which is there). This technique works perfectly for several images even when the objects are translated, scaled

In order to further justify this work, the descriptors of numbers are matched with each other using AMDF. The results are found to be similar to that of polygon descriptors which is shown in table 3 . Here too, the diagonal elements are found to be zero (ideal case).

\begin{tabular}{|l|l|l|l|l|l|l|l|l|l|l|}
\hline & $\mathbf{1}$ & $\mathbf{2}$ & $\mathbf{3}$ & $\mathbf{4}$ & $\mathbf{5}$ & $\mathbf{6}$ & $\mathbf{7}$ & $\mathbf{8}$ & $\mathbf{9}$ & $\mathbf{0}$ \\
\hline $\mathbf{1}$ & 0 & 0.07 & 0.14 & 0.23 & 0.17 & 0.22 & 0.1 & 0.2 & 0.18 & 0.23 \\
\hline $\mathbf{2}$ & 0.07 & 0 & 0.09 & 0.26 & 0.13 & 0.19 & 0.1 & 0.2 & 0.17 & 0.17 \\
\hline $\mathbf{3}$ & 0.14 & 0.09 & 0 & 0.24 & 0.08 & 0.10 & 0.1 & 0.1 & 0.11 & 0.12 \\
\hline $\mathbf{4}$ & 0.23 & 0.26 & 0.24 & 0 & 0.28 & 0.24 & 0.2 & 0.2 & 0.22 & 0.17 \\
\hline $\mathbf{5}$ & 0.17 & 0.13 & 0.08 & 0.28 & 0 & 0.10 & 0.1 & 0.1 & 0.16 & 0.17 \\
\hline $\mathbf{6}$ & 0.22 & 0.19 & 0.10 & 0.24 & 0.10 & 0 & 0.2 & 0.1 & 0.16 & 0.09 \\
\hline $\mathbf{7}$ & 0.16 & 0.13 & 0.15 & 0.23 & 0.13 & 0.21 & 0 & 0.2 & 0.22 & 0.2 \\
\hline $\mathbf{8}$ & 0.21 & 0.18 & 0.12 & 0.20 & 0.17 & 0.11 & 0.2 & 0.1 & 0.10 & 0.04 \\
\hline $\mathbf{9}$ & 0.18 & 0.17 & 0.11 & 0.22 & 0.16 & 0.16 & 0.2 & 0.1 & 0 & 0.1 \\
\hline $\mathbf{0}$ & 0.23 & 0.17 & 0.12 & 0.17 & 0.17 & 0.09 & 0.2 & 0.1 & 0.1 & 0 \\
\hline
\end{tabular}

Table 3: AMDF coefficient of number descriptors

\section{RESULTS AND CONCLUSIONS}

The descriptors are found to be unique for both polygons and numbers. The AMDF technique works quite better than the similarity measure ACF in many applications, sometimes ACF fails even when two same objects are compared. It is clearly seen in the table 1 and 2 that even when same object's characteristics are compared with each other, the ACF is a failure in this case. Area filtering is done which is sufficient for noise invariance. The idea is to make descriptors easy, simple and computationally efficient. Further work is carried out to make the descriptor rotation invariant as well as finding descriptors for all alphabets so that a simple technique for optical character recognition is found. Since this technique does not require any floating point calculations (unlike Fourier descriptors), it is easy to implement in hardware. Also, the memory requirement is also very less. Moreover, this technique is computationally efficient unlike moment invariant techniques.

\section{ACKNOWLEDGEMENT}

We would like to thank Prof. Rabinder Henry and Prof. Amit Patwardhan for guiding us and showing the true value of research.
We would like to thank all their friends who have helped them whatever way they can.

\section{REFERENCES}

[1] Mark S. Nixon, Alberto S. Aguado, Feature Extraction and Image Processing, Chapter 7.page 247-248.

[2] Anil K. Jain,Fellow,IEEE, Robert P.W.Duin and Jianchang Mao.Senior Member IEEE, Statistical Pattern Recognition: A review, IEEE transaction on pattern analysis.,vol 22.

[3] El-Bakry, H.M. Abo Elsoud, M.A, Human face recognition using neural networks. Radio Science Conference, 1999. NRSC ' 99. Proceedings of the Sixteenth National.

[4] David Boulinguez and Andre Quinquis, Underwater Burried object recognition using Wavelet Packets and Fourier Descriptors.

[5] Akrem El-ghazal, Otman Basir, Saeid Belkasim, Farthest point distance: A new shape signature for Fourier descriptors. Signal Processing: Image Communication 24 (2009) 572-586

[6] A. Ashbrook and N.A.Thacker, Tutorial: Algorithms For 2Dimensional Object Recognition.

[7] Earl Gose , Richard Johnson Baugh and Steve Jost, Pattern recognition and Image Analysis. page 397

[8] Mikhail Lange, Sergey Ganebnykh and Andrey Lange, Moment-Based Pattern Representation Using Shape and Grayscale Features.

[9] Jan Flusser, Tomas Suk, Character Recognition by Affine Moment Invariants.

[10] C.H. Teh, R.T. Chin, On image analysis by the methods of moments, IEEE Trans. Pattern Analysis. Machine Intelligence.

[11] Otsu, N., "A Threshold Selection Method from Gray-Level Histograms," IEEE Transactions on Systems, Man, and Cybernetics, Vol. 9, No. 1, 1979, pp. 62-66.

[12] Sonka, Hlavac ,Boyle,Digital Image Processing and Computer Vision.

[13] Haralick, Robert M., and Linda G. Shapiro, Computer and Robot Vision, Volume I, Addison-Wesley, 1992, pp. 28-48.

[14] Rafael C. Gonzalez, Richard E. Woods, Digital Image Processing, chapter 9. 